Research Article

\title{
The Delayed Doubly Stochastic Linear Quadratic Optimal Control Problem
}

\author{
Yan Chen ${ }^{1,2}$ and Jie $\mathrm{Xu} \mathbb{( D D}^{3}$ \\ ${ }^{1}$ Department of Mathematics, Jilin University, Changchun 130012, China \\ ${ }^{2}$ School of Mathematics, Changchun Normal University, Changchun 130032, China \\ ${ }^{3}$ College of Science, Jilin Institute of Chemical Technology, Jilin 132022, China
}

Correspondence should be addressed to Jie Xu; aqie990132@126.com

Received 9 April 2020; Accepted 14 May 2020; Published 6 June 2020

Guest Editor: Wenguang Yu

Copyright ( 92020 Yan Chen and Jie Xu. This is an open access article distributed under the Creative Commons Attribution License, which permits unrestricted use, distribution, and reproduction in any medium, provided the original work is properly cited.

In this paper, the delayed doubly stochastic linear quadratic optimal control problem is discussed. It deduces the expression of the optimal control for the general delayed doubly stochastic control system which contained time delay both in the state variable and in the control variable at the same time and proves its uniqueness by using the classical parallelogram rule. The paper is concerned with the generalized matrix value Riccati equation for a special delayed doubly stochastic linear quadratic control system and aims to give the expression of optimal control and value function by the solution of the Riccati equation.

\section{Introduction}

As is known to all, the stochastic differential equation and stochastic analysis have developed rapidly. The theory of the stochastic differential equation is widely used in economy, biology, physics, financial mathematics, and other fields. The latest research on the insurance model was given in $[1-3]$. The social optimal mean field control problem was discussed in [4]. In order to provide a probabilistic interpretation for the solution of a kind of partial differential equations, Pardoux and Peng [5] first introduced the backward doubly stochastic differential equations and proved the existence and uniqueness of this kind of differential. Then, people began to study doubly stochastic differential equations. Zhu and Shi $[6,7]$ were concerned with a class of partial information control problems for backward doubly stochastic systems and gave the maximum principle and its applications for the system. Recently, Shi and Zhu [8] studied a type of forwardbackward doubly stochastic differential equations driven by Brownian motions and the Poisson process and applied the result to backward doubly stochastic linear quadratic nonzero sum differential games with random jumps to get the explicit form of the open-loop Nash equilibrium point by the solution of this kind of equation. With the deepening of research, people gradually realized that many problems are not only affected by the current situation, but also by their past history. This kind of problem is called the delay problem. The equation describing this kind of problem is called the delay equation. Due to the fact that time delay widely exists in the practical systems, it will cause the change in system performance. Therefore, it can increase the control difficulty of the system. Delayed problems have become the focus of scholar's research studies. Chen and $\mathrm{Wu}$ [9] considered the delayed backward system and obtained the maximum principle for these problems. Wu and Wang [10] studied the optimal control problem of the backward stochastic differential delay equation under partial information. Lv et al. [11] considered the maximum principle for optimal control of anticipated forwardbackward stochastic delayed systems with regime switching. Wang and $\mathrm{Wu}$ [12] were concerned with the optimal control problems of forward-backward delay systems involving impulse controls and established the stochastic maximum principle for this kind of systems. Yu [13] investigated the maximum principle for stochastic optimal control problems of delay systems with random coefficients involving both continuous and impulse controls. 
Linear quadratic (LQ) optimal control problem is the theoretical basis for many problems. When delay variables exist in the doubly stochastic control system, the LQ problem becomes more complex and interesting. Chen and $\mathrm{Wu}$ [14] considered the LQ problem with delay in which the state depended on the past time but not the control in the system. Tang and $\mathrm{Wu}$ [15] were concerned with the linear stochastic system with Lévy processes. Huang et al. [16] were concerned with one kind of delayed forward-backward linear quadratic stochastic control problems and derived the explicit form of the optimal control. However, to our best knowledge, there is little work on the doubly stochastic LQ problem with delay. Based on the abundant literature, we want to discuss the delayed doubly stochastic LQ problem. When the LQ control system contains time delay, some important characteristic changes have taken place in research. The system contains a delayed doubly stochastic differential equation and a new kind of equation called the anticipated backward doubly stochastic differential equation which was discussed in $[17,18]$. Inspired by the idea of the maximum principle for the delayed doubly stochastic control system $[19,20]$, we studied the general LQ system in which both the state variable and the control variable contain time delay at the same time. As is known to all, it is the key to find out the feedback control of the LQ problem. We deduce the explicit expression of the optimal control for the delayed doubly stochastic LQ problem. We consider the matrix Riccati equation for a class of the LQ problem. We deduced the solution of the LQ system by the solution corresponding to the Riccati equation, which was introduced originally by Peng [21]. We hope that our research can better describe the optimal feedback control of the delayed doubly stochastic LQ problem.

The rest of our paper is organized as follows. First, we introduce preliminary results and some necessary notations. In Section 3, we give the explicit expression of optimal control and prove its uniqueness by using the classical parallelogram rule. And then, we discuss a special kind of the control system, in which the time delay is contained only in the control variables. We try to introduce the generalized matrix value Riccati equation corresponding to the system. And then, we use the solution of the Riccati equation to show the optimal control for the delayed doubly stochastic LQ problem. At the same time, we indicate the objective function by the solution of the Riccati equation and the initial value of the state variable.

\section{Preliminaries}

First, let us introduce the common notations in this paper. Let $(\Omega, \mathscr{F}, P)$ be a probability space. Assume $\{W(t): 0 \leq t \leq T\}$ and $\{B(t): 0 \leq t \leq T\}$ be two mutually independent standard Brownian motions defined on $(\Omega, \mathscr{F}, P)$, with values, respectively, in $R^{m}$ and in $R^{d}$. Note the integral with respect to $\{W(t)\}$ as the forward Itô's integral and $\{B(t)\}$ as the backward Itô's integral. Let $\mathrm{N}$ denote the class of $P$ null sets of $\mathscr{F}$. We define $\mathscr{F}_{t}^{w}=\mathrm{N} \vee \sigma\{W(r)-W(0): 0 \leq r \leq t\}$,

$\mathscr{F}_{t, T}^{B}=\mathrm{N} \vee \sigma\{B(r)-B(t): t \leq r \leq T\}$, and $\mathscr{F}_{t}=\mathscr{F}_{t}^{w} \vee \mathscr{F}_{t, T}^{B}$ for each $t \in[0, T]$. We denote $M^{2}\left(0, T ; R^{n}\right)$ the set of all classes of $(\mathrm{d} t \times \mathrm{d} P$ a.e. equal $) \mathscr{F}_{t}$ measurable stochastic process $\varphi(t)$ satisfying $E \int_{0}^{T}|\varphi(t)|^{2} \mathrm{~d} t<+\infty$. Similarly, $S^{2}\left(0, T ; R^{n}\right)$ denotes the set of continuous $n$-dimensional $\mathscr{F}_{t}$ measurable stochastic process $\varphi(t)$ satisfying $\operatorname{Esup}_{t \in[0, T]}|\varphi(t)|^{2}<+\infty$. $E^{\mathscr{F}_{t}}[\cdot]=E\left[\mathscr{F}_{t}\right]$ denotes the conditional expectation under filtration $\mathscr{F}_{t} \cdot\langle\cdot, \cdot\rangle$ denotes the scalar product, and $T$ in the superscripts means the transpose of the matrix.

In this paper, we mainly investigate the delayed doubly stochastic linear quadratic control system:

$$
\left\{\begin{array}{l}
\mathrm{d} x(t)=\left[A_{1}(t) x(t)+B_{1}(t) x_{\delta}(t)+C_{1}(t) y(t)+D_{1}(t) y_{\delta}(t)+E_{1}(t) u(t)+F_{1}(t) u_{\delta}(t)\right] \mathrm{d} t \\
+\left[A_{2}(t) x(t)+B_{2}(t) x_{\delta}(t)+C_{2}(t) y(t)+D_{2}(t) y_{\delta}(t)+E_{2}(t) u(t)+F_{2}(t) u_{\delta}(t)\right] \\
\overrightarrow{\mathrm{d} W(t)}-y(t) \mathrm{d} \overleftrightarrow{B}(t), \quad t \in[0, T] \\
x(t)=\varphi(t), \quad t \in[-\delta, 0] \\
y(t)=\psi(t), \quad t \in[-\delta, 0] \\
u(t)=0, \quad t \in[-\delta, 0]
\end{array}\right.
$$

where the notation $x_{\delta}(t)=x(t-\delta), y_{\delta}(t)=y(t-\delta)$, and $u_{\delta}(t)=u(t-\delta)$.

Remark 1. In this delayed doubly stochastic control system, the state and control variables contain time delay at the same time. Time delay exists all the time in the system. But, we do nothing before the initial time. So, we give the assumption that $u(t)=0$ when time $t$ belongs to the interval before the control intervenes.

The cost functional is written as

$$
J(u(\cdot))=\frac{1}{2} E\left\{\int_{0}^{T}[\langle K(t) x(t), x(t)\rangle+\langle R(t) y(t), y(t)\rangle+\langle S(t) u(t), u(t)\rangle] \mathrm{d} t+\langle Q x(T), x(T)\rangle\right\} .
$$


For a convex subset $U \subset R^{k}$, we define $U[0, T]$ as follows:

$\left.U[0, T]:=\left\{u:[0, T] \times \Omega \longrightarrow U, E \int_{0}^{T}|u(t)|^{2} \mathrm{~d} t<+\infty\right\}\right\}$.

$$
J\left(u^{*}(\cdot)\right)=\inf _{u(\cdot) \in U[0, T]} J(u(\cdot)),
$$

the corresponding $\left(x^{*}(\cdot), y^{*}(\cdot), u^{*}(\cdot)\right)$ is called an optimal triple.

The corresponding adjoint equation becomes

Our optimal control problem can be stated as minimizing the cost functional over $U[0, T]$. For optimal control $u^{*}(\cdot)$ satisfying

$$
\left\{\begin{array}{l}
-\mathrm{d} p(t)=\left\{A_{1}^{\top}(t) p(t)+E^{\mathscr{F}_{t}}\left[B_{1}^{\top}(t+\delta) p(t+\delta)\right]+A_{2}^{\top}(t) q(t)-K(t) x(t)+E^{\mathscr{F}_{t}}\left[B_{2}^{\top}(t+\delta) q(t+\delta)\right]\right\} \mathrm{d} t \\
+\left\{R(t) y(t)-C_{1}^{\top}(t) p(t)-C_{2}^{\top}(t) q(t)-E^{\mathscr{F}_{t}}\left[D_{1}^{\top}(t+\delta) p(t+\delta)\right]-E^{\mathscr{F}_{t}}\left[D_{2}^{\top}(t+\delta) q(t+\delta)\right]\right\} \mathrm{d} \overleftarrow{B}(t), \\
-q(t) \overrightarrow{\mathrm{d} W(t)}, \quad t \in[0, T], \\
p(T)=-Q x(T), \\
p(t)=0, \quad t \in(T, T+\delta], \\
q(t)=0, \quad t \in(T, T+\delta] .
\end{array}\right.
$$

We assume that the following conditions hold:

(A1) Assume that the coefficient matrices $A_{i}, B_{i}, C_{i}, D_{i}$, $E_{i}$, and $F_{i}(i=1,2)$ are the matrix process with a proper dimension

(A2) The random matrix $Q: \Omega \longrightarrow R^{n \times n}$ is the nonnegatively bounded symmetric $\mathscr{F}_{t}$ adapt matrix

(A3) All the functions of $t$ are bounded, $K(t), R(t)$, and $Q$ are symmetric nonnegative definite, and $S(t)$ is symmetric uniformly positive definite

$$
\begin{aligned}
u^{*}(t)= & S^{-1}(t)\left\{E_{1}^{\top}(t) p(t)+E_{2}^{\top}(t) q(t)\right. \\
& \left.+E^{\mathscr{F}_{t}}\left[F_{1}^{\top}(t+\delta) p(t+\delta)+F_{2}^{\top}(t+\delta) q(t+\delta)\right]\right\}
\end{aligned}
$$

$t \in[0, T]$, is the unique optimal control for the delayed doubly stochastic linear quadratic optimal control problem, where $\left(x^{*}(\cdot), y^{*}(\cdot), p(\cdot), q(\cdot)\right)$ is the solution of the following system:

\section{Main Results}

Theorem 1. The function

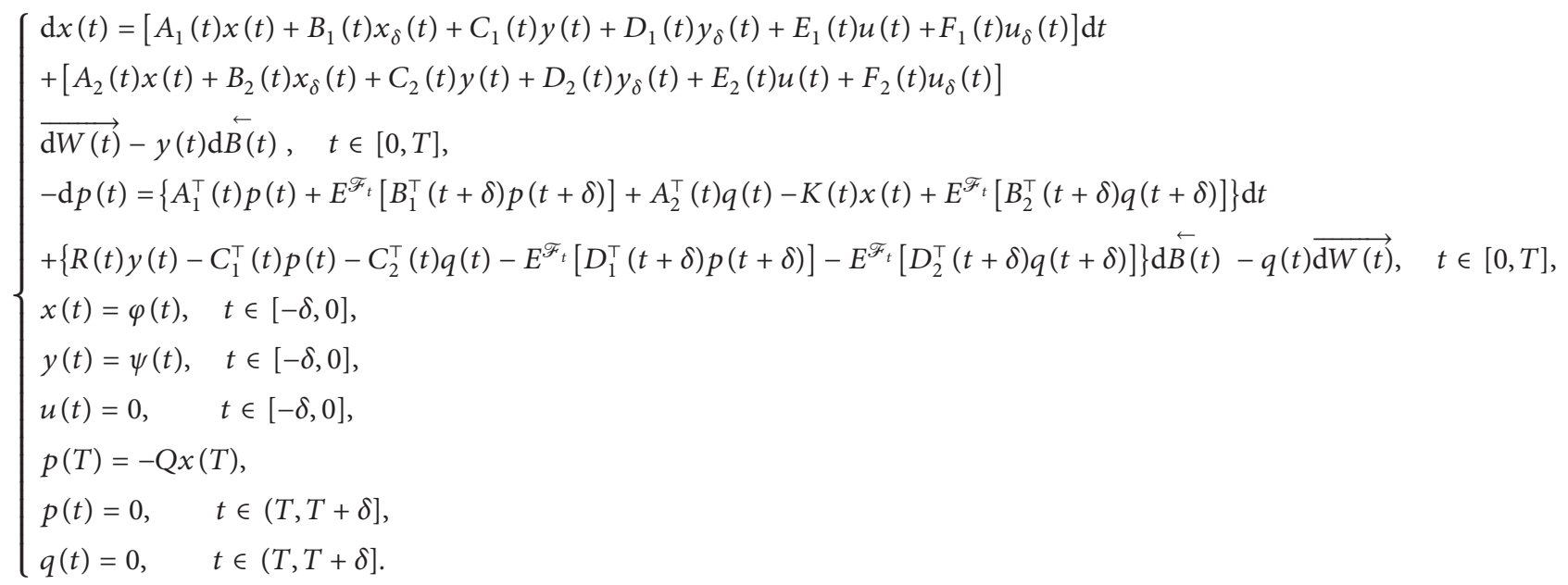


Proof. The existence and uniqueness of the solution for equation (1) can be guaranteed by Theorem 3.1 in [20] under the assumptions (A1)-(A3). Equation (5) is an anticipated backward doubly stochastic differential equation. Its existence and uniqueness can be deduced by Theorem 3.2 in
[18]. Now, we prove that $u^{*}(t)$ is the optimal control. For all $v(\cdot) \in U[0, T]$, let $\left(x^{*}(\cdot), y^{*}(\cdot)\right)$ and $\left(x^{v}(\cdot), y^{v}(\cdot)\right)$ be the trajectory of the system corresponding to $u^{*}(t)$ and $v(t)$, respectively.

Then,

$$
\begin{aligned}
J( & v(\cdot))-J\left(u^{*}(\cdot)\right) \\
= & \frac{1}{2} E\left[\int _ { 0 } ^ { T } \left(\left\langle K(t) x^{v}(t), x^{v}(t)\right\rangle-\left\langle K(t) x^{*}(t), x^{*}(t)\right\rangle+\left\langle R(t) y^{v}(t), y^{v}(t)\right\rangle\right.\right. \\
& \left.-\left\langle R(t) y^{*}(t), y^{*}\right\rangle+\langle S(t) v(t), v(t)\rangle-\left\langle S(t) u^{*}(t), u^{*}(t)\right\rangle\right) \mathrm{d} t \\
& \left.+\left\langle Q x^{v}(T), x^{v}(T)\right\rangle-\left\langle Q x^{*}(T), x^{*}(T)\right\rangle\right], \\
= & \frac{1}{2} E\left[\int _ { 0 } ^ { T } \left(\left\langle K(t)\left(x^{v}(t)-x^{*}(t)\right), x^{v}(t)-x^{*}(t)\right\rangle+\left\langle S(t)\left(v(t)-u^{*}(t)\right), v(t)-u^{*}(t)\right\rangle\right.\right. \\
& +\left\langle R(t)\left(y^{v}(t)-y^{*}(t)\right), y^{v}(t)-y^{*}(t)\right\rangle+2\left\langle K(t) x^{*}(t), x^{v}(t)-x^{*}(t)\right\rangle \\
& \left.+2\left\langle R(t) y^{*}(t), y^{v}(t)-y^{*}(t)\right\rangle+2\left\langle S(t) u^{*}(t), v(t)-u^{*}(t)\right\rangle\right) \mathrm{d} t \\
& \left.+\left\langle Q\left(x^{v}(T)-x^{*}(T)\right), x^{v}(T)-x^{*}(T)\right\rangle+2\left\langle Q x^{*}(T), x^{v}(T)-x^{*}(T)\right\rangle\right] .
\end{aligned}
$$

From the definitions of $K(t), R(t), S(t)$, and $Q$, we know $K(t), R(t)$, and $Q$ are symmetric nonnegative definite and $S(t)$ is symmetric uniformly positive definite. So, we have

$J(v(\cdot))-J\left(u^{*}(\cdot)\right)$

$\geq E\left\{\int_{0}^{T}\left[\left\langle K(t) x^{*}(t), x^{v}(t)-x^{*}(t)\right\rangle+\left\langle S(t) u^{*}(t), v(t)-u^{*}(t)\right\rangle\right.\right.$

$\left.\left.+\left\langle R(t) y^{*}(t), y^{v}(t)-y^{*}(t)\right\rangle\right] \mathrm{d} t+\left\langle Q x^{*}(T), x^{v}(T)-x^{*}(T)\right\rangle\right\}$.
Applying the Itô-Doeblin formula to

$$
\left\langle Q x^{*}(T), x^{v}(T)-x^{*}(T)\right\rangle=\left\langle-p(T), x^{v}(T)-x^{*}(T)\right\rangle,
$$

and paying attention to the initial condition and the terminal condition, we have

$$
\begin{aligned}
E & \left\langle p(T), x^{v}(T)-x^{*}(T)\right\rangle \\
= & E\left[\int _ { 0 } ^ { T } \left\langlep(t), A_{1}(t)\left(x^{v}(t)-x^{*}(t)\right)+B_{1}(t)\left(x_{\delta}^{v}(t)-x_{\delta}^{*}(t)\right)+C_{1}(t)\left(y^{v}(t)-y^{*}(t)\right)\right.\right. \\
& \left.+D_{1}(t)\left(y_{\delta}^{v}(t)-y_{\delta}^{*}(t)\right)+E_{1}(t)\left(v(t)-u^{*}(t)\right)+F_{1}(t)\left(v_{\delta}(t)-u_{\delta}^{*}(t)\right)\right\rangle \mathrm{d} t \\
& -\int_{0}^{T}\left\langle A_{1}^{\top}(t) p(t)+A_{2}^{\top}(t) q(t)-K(t) x^{*}(t)+E^{\mathscr{F}} t\left[B_{1}^{\top}(t+\delta) p(t+\delta)\right]\right. \\
& \left.+E^{\mathscr{F}_{t}}\left[B_{2}^{\top}(t+\delta) q(t+\delta)\right], x^{v}(t)-x^{*}(t)\right\rangle \mathrm{d} t \\
& +\int_{0}^{T}\left\langle q(t), A_{2}(t)\left(x^{v}(t)-x^{*}(t)\right)+B_{2}(t)\left(x_{\delta}^{v}(t)-x_{\delta}^{*}(t)\right)+C_{2}(t)\left(y^{v}(t)-y^{*}(t)\right)\right. \\
& \left.+D_{2}(t)\left(y_{\delta}^{v}(t)-y_{\delta}^{*}(t)\right)+E_{2}(t)\left(v(t)-u^{*}(t)\right)+F_{2}(t)\left(v_{\delta}(t)-u_{\delta}^{*}(t)\right)\right\rangle \mathrm{d} t \\
& +\int_{0}^{T}\left\langle R(t) y^{*}(t)-C_{1}^{\top}(t) p(t)-C_{2}^{\top}(t) q(t)-E^{\mathscr{F}_{t}}\left[D_{1}^{\top}(t+\delta) p(t+\delta)\right]\right. \\
& \left.\left.-E^{\mathscr{F}}{ }^{\top}\left[D_{2}^{\top}(t+\delta) q(t+\delta)\right], y^{v}(t)-y^{*}(t)\right\rangle \mathrm{d} t\right] .
\end{aligned}
$$


In fact, we have

$$
\begin{aligned}
& E \int_{0}^{T}\left[\left\langle p(t), B_{1}(t)\left(x_{\delta}^{v}(t)-x_{\delta}^{*}(t)\right)\right\rangle-\left\langle E^{\mathscr{F}_{t}}\left[B_{1}^{\top}(t+\delta) p(t+\delta)\right], x^{v}(t)-x^{*}(t)\right\rangle\right] \mathrm{d} t \\
& =E \int_{0}^{T}\left\langle p(t), B_{1}(t)\left(x_{\delta}^{v}(t)-x_{\delta}^{*}(t)\right)\right\rangle \mathrm{d} t-\int_{\delta}^{T+\delta}\left\langle B_{1}^{\top}(t) p(t), x_{\delta}^{v}(t)-x_{\delta}^{*}(t)\right\rangle \mathrm{d} t \\
& =E \int_{0}^{\delta}\left\langle p(t), B_{1}(t)\left(x_{\delta}^{v}(t)-x_{\delta}^{*}(t)\right)\right\rangle \mathrm{d} t-\int_{T}^{T+\delta}\left\langle B_{1}^{\top}(t) p(t), x_{\delta}^{v}(t)-x_{\delta}^{*}(t)\right\rangle \mathrm{d} t \\
& =0 .
\end{aligned}
$$

So, we have

$$
\begin{aligned}
E\left\langle-p(T), x^{v}(T)-x^{*}(T)\right\rangle \\
=E \int_{0}^{T}\left[\left\langle-K(t) x^{*}(t), x^{v}(t)-x^{*}(t)\right\rangle+\left\langle-R(t) y^{*}(t), y^{v}(t)-y^{*}(t)\right\rangle\right. \\
\quad+\left\langle-p(t), E_{1}(t)\left(v(t)-u^{*}(t)\right)+F_{1}(t)\left(v_{\delta}(t)-u_{\delta}^{*}(t)\right)\right\rangle \\
\left.\quad+\left\langle-q(t), E_{2}(t)\left(v(t)-u^{*}(t)\right)+F_{2}(t)\left(v_{\delta}(t)-u_{\delta}^{*}(t)\right)\right\rangle\right] \mathrm{d} t .
\end{aligned}
$$

Then,

$$
\begin{aligned}
& J(v(\cdot))-J\left(u^{*}(\cdot)\right) \geq E \int_{0}^{T}\left[\left\langle S(t) u^{*}(t), v(t)-u^{*}(t)\right\rangle+\left\langle-p(t), E_{1}(t)\left(v(t)-u^{*}(t)\right)\right.\right. \\
& \left.\quad+F_{1}(t)\left(v_{\delta}(t)-u_{\delta}^{*}(t)\right)\right\rangle+\left\langle-q(t), E_{2}(t)\left(v(t)-u^{*}(t)\right)\right. \\
& \left.\left.\quad+F_{2}(t)\left(v_{\delta}(t)-u_{\delta}^{*}(t)\right)\right\rangle\right] \mathrm{d} t
\end{aligned}
$$

So from the definition of $u^{*}(t)$, we have

$$
J(v(\cdot))-J\left(u^{*}(\cdot)\right) \geq 0
$$

for any $v(\cdot) \in U[0, T]$. This shows that $u^{*}(t)$ is the optimal control.

Next, we will prove the uniqueness. Assume that $u_{1}(\cdot)$ and $u_{2}(\cdot)$ are both optimal controls. $\left(x_{1}(\cdot), y_{1}(\cdot)\right)$ and $\left(x_{2}(\cdot), y_{2}(\cdot)\right)$ are the trajectories corresponding to $u_{1}(\cdot)$ and $u_{2}(\cdot)$, respectively. Equation (5) is a new type of the anticipated backward doubly stochastic differential equation. The existence and uniqueness of the solution for the equation can be guaranteed by Theorem 3.2 in [18]. By the uniqueness of the solution of the equation, we know that $\left(\left(x_{1}(\cdot)+x_{2}(\cdot) / 2\right),\left(y_{1}(\cdot)+y_{2}(\cdot) / 2\right)\right)$ is the trajectory corresponding to $\left(u_{1}(\cdot)+u_{2}(\cdot) / 2\right)$. From the definition of $K(t), \quad R(t), \quad S(t)$, and $Q$, we know $J\left(u^{1}(\cdot)\right)=$ $J\left(u^{2}(\cdot)\right)=\alpha \geq 0$.

Then,

$$
2 \alpha=J\left(u_{1}(\cdot)\right)+J\left(u_{2}(\cdot)\right)
$$

$$
\begin{aligned}
= & J\left(\frac{u_{1}(\cdot)+u_{2}(\cdot)}{2}\right) \\
& +E \int_{0}^{T}\left[\left\langle S(t) \frac{u_{1}(t)-u_{2}(t)}{2}, \frac{u_{1}(t)-u_{2}(t)}{2}\right\rangle\right. \\
& +\left\langle K(t) \frac{x_{1}(t)-x_{2}(t)}{2}, \frac{x_{1}(t)-x_{2}(t)}{2}\right\rangle \\
& \left.+\left\langle R(t) \frac{y_{1}(t)-y_{2}(t)}{2}, \frac{y_{1}(t)-y_{2}(t)}{2}\right\rangle\right] \mathrm{d} t \\
& +\left\langle Q \frac{x_{1}(T)-x_{2}(T)}{2}, \frac{x_{1}(T)-x_{2}(T)}{2}\right\rangle \\
\geq & 2 \alpha+E \int_{0}^{T}\left\langle S(t) \frac{u_{1}(t)-u_{2}(t)}{2}, \frac{u_{1}(t)-u_{2}(t)}{2}\right\rangle \mathrm{d} t .
\end{aligned}
$$


From the definition of $S(t)$, we have $u_{1}(\cdot)=u_{2}(\cdot)$. We complete the proof of Theorem 1.

Next, we study a special class of the delayed doubly stochastic LQ problem. We discuss the case that only the control contained the delayed variable, and the initial value of the state variable $\eta$ is deterministic. The delayed system can be written as

$$
\left\{\begin{array}{l}
\mathrm{d} x(t)=\left[A_{1}(t) x(t)+C_{1}(t) y(t)+F_{1}(t) u_{\delta}(t)\right] \mathrm{d} t+\left[A_{2}(t) x(t)\right. \\
\left.+C_{2}(t) y(t)+F_{2}(t) u_{\delta}(t)\right] \overrightarrow{\mathrm{d} W(t)}-y(t) \mathrm{d} \overleftrightarrow{B(t)}, \quad t \in[0, T], \\
-\mathrm{d} p(t)=\left[A_{1}^{\top}(t) p(t)+A_{2}^{\top}(t) q(t)-K(t) x(t)\right] \mathrm{d} t+[R(t) y(t) \\
\left.-C_{1}^{\top}(t) p(t)-C_{2}^{\top}(t) q(t)\right] \mathrm{d} \overleftarrow{B(}(t)-q(t) \overrightarrow{\mathrm{d} W(t)}, \quad t \in[0, T], \\
x(0)=\eta, \\
u(t)=0, \quad t \in[-\delta, 0], \\
p(T)=-Q x(T), \\
p(t)=0, \quad t \in(T, T+\delta], \\
q(t)=0, \quad t \in(T, T+\delta] .
\end{array}\right.
$$

We also consider the optimal control problem (4) under the classical quadratic index (4).

From Theorem 1, we can deduce the optimal control directly:

$$
\begin{array}{r}
u^{*}(t)=S^{-1}(t) E^{\mathscr{F}} t\left[F_{1}^{\top}(t+\delta) p(t+\delta)+F_{2}^{\top}(t+\delta) q(t+\delta)\right] \\
t \in[0, T] .
\end{array}
$$

Peng [21] gave the solution of a kind of stochastic Hamiltonian systems by the Riccati equation corresponding to the system. Following this way, we want to use the solution of the Riccati equation to introduce the optimal control for the delayed doubly stochastic LQ problem. First, we give the generalized matrix Riccati equation:

$$
\left\{\begin{array}{l}
-\dot{M}(t)=M(t) A_{1}(t)+A_{1}^{\top}(t) M(t)+M(t) C_{1}(t) G(t)+A_{2}^{\top}(t) N(t) \\
-K(t)+M(t) F_{1}(t) S^{-1}(t-\delta)\left[F_{1}^{\top}(t) M(t)+F_{2}^{\top}(t) N(t)\right] \\
M(t) G(t)=R(t) G(t)-C_{1}^{\top}(t) M(t)-C_{2}^{\top}(t) N(t), \\
N(t)=M(t) A_{2}(t)+M(t) F_{2}(t) S^{-1}(t-\delta)\left[F_{1}^{\top}(t) M(t)+F_{2}^{\top}(t) N(t)\right] \\
+M(t) C_{2}(t) G(t) .
\end{array}\right.
$$

Theorem 2. Let the assumptions (A1)-(A3) be satisfied. If the Riccati equation has a solution $(G(\cdot), M(\cdot), N(\cdot))$, then system (17) has a unique solution:

$$
(x(t), y(t), p(t), q(t))=(x(t), G(t) x(t), M(t) x(t), N(t) x(t)) \text {, }
$$

where $x(t)$ is solved by

$$
\left\{\begin{array}{l}
\mathrm{d} x(t)=\left\{A_{1}(t)+C_{1}(t) G(t)+F_{1}(t) S^{-1}(t-\delta) E^{\mathscr{F}_{t-\delta}}\left[F_{1}^{\top}(t) p(t)\right.\right. \\
\left.\left.+F_{2}^{\top}(t) q(t)\right]\right\} x(t) \mathrm{d} t+\left\{A_{2}(t)+C_{2}(t) G(t)+F_{2}(t) S^{-1}(t-\delta)\right. \\
\left.E^{\mathscr{F}_{t-\delta}}\left[F_{1}^{\top}(t) p(t)+F_{2}^{\top}(t) q(t)\right]\right\} x(t) \overrightarrow{\mathrm{d} W(t)}-y(t) \mathrm{d} \overleftrightarrow{B}(t), \quad t \in[0, T] \\
x(0)=\eta, \\
u(t)=0, \quad t \in[-\delta, 0] .
\end{array}\right.
$$

Proof. We apply Itô's formula to $M(t) x(t)$ and compare the coefficient with $p(t)$, then we can deduce the conclusion directly. Next, we will prove the uniqueness.
Assume that $(x(t), y(t), p(t), q(t))$ is a solution of system (17), with the conditions $x(0)=\eta$ and $P(T)=-Q x(T)$. 
We set $\quad \bar{y}(t)=G(t) x(t), \quad \bar{p}(t)=M(t) x(t), \quad$ and $\bar{q}(t)=N(t) x(t)$. By differentiating $\bar{p}(t)$, we have

$$
\begin{aligned}
\mathrm{d} \bar{p}(t)= & \dot{M}(t) x(t) \mathrm{d} t+M(t) \mathrm{d} x(t) \\
= & \dot{M}(t) x(t) \mathrm{d} t+M(t)\left[A_{1}(t) x(t)+C_{1}(t) y(t)+F_{1}(t) u_{\delta}(t)\right] \mathrm{d} t \\
& +M(t)\left[A_{2}(t) x(t)+C_{2}(t) y(t)+F_{2}(t) u_{\delta}(t)\right] \overrightarrow{\mathrm{d} W(t)}-M(t) y(t) \mathrm{d} \overleftrightarrow{\leftarrow}(t) .
\end{aligned}
$$

Substituting the first and the second equality of the Riccati equation (19) into (22), we have

$$
\begin{aligned}
\mathrm{d} \bar{p}(t)= & -\left[A_{1}^{\top}(t) \bar{p}(t)+M(t) C_{1}(t)(\bar{y}(t)-y(t))+A_{2}^{\top}(t) \bar{q}(t)-K(t) x(t)\right] \mathrm{d} t \\
& +\left[\bar{q}(t)-M(t) C_{2}(t)(\bar{y}(t)-y(t))\right] \overrightarrow{\mathrm{d} W(t)}-M(t) y(t) \mathrm{d} \overleftrightarrow{B_{(}(t)} .
\end{aligned}
$$

On the other hand, from the Riccati equation (19), we have

$$
\begin{aligned}
& M(t) y(t)=M(t)\{\bar{y}(t)-[\bar{y}(t)-y(t)]\} \\
& =M(t) G(t) x(t)-M(t)[\bar{y}(t)-y(t)] \\
& =\left[R(t) G(t)-C_{1}^{\top}(t) M(t)-C_{2}^{\top}(t) N(t)\right] x(t)-M(t)[\bar{y}(t)-y(t)] \\
& =R(t) \bar{y}(t)-C_{1}^{\top}(t) \bar{p}(t)-C_{2}^{\top}(t) \bar{q}(t)-M(t)[\bar{y}(t)-y(t)] .
\end{aligned}
$$

$$
\begin{aligned}
\mathrm{d} \bar{p}(t)= & -\left[A_{1}^{\top}(t) \bar{p}(t)+M(t) C_{1}(t)(\bar{y}(t)-y(t))+A_{2}^{\top}(t) \bar{q}(t)-K(t) x(t)\right] \mathrm{d} t \\
& +\left[\bar{q}(t)-M(t) C_{2}(t)(\bar{y}(t)-y(t))\right] \overline{\mathrm{d} W(t)} \\
& -\left[R(t) \bar{y}(t)-C_{1}^{\top}(t) \bar{p}(t)-C_{2}^{\top}(t) \bar{q}(t)-M(t)(\bar{y}(t)-y(t))\right] \mathrm{d} \overleftarrow{B(}(t) .
\end{aligned}
$$

We denote $(\hat{y}(t), \widehat{p}(t), \widehat{q}(t))=(\bar{y}(t)-y(t), \bar{p}(t)-$ $p(t), \bar{q}(t)-q(t))$, then we have

$-\mathrm{d} \widehat{p}(t)=-\mathrm{d} \bar{p}(t)-(-\mathrm{d} p(t))$

$=\left[A_{1}^{\top}(t) \widehat{p}(t)+A_{2}^{\top}(t) \widehat{q}(t)+M(t) C_{1}(t) \widehat{y}(t)\right] \mathrm{d} t$

$+\left[R(t) \widehat{y}(t)-C_{1}^{\top}(t) \widehat{p}(t)-C_{2}^{\top}(t) \widehat{q}(t)-M(t) \widehat{y}(t)\right] \mathrm{d} \overleftarrow{B}(t)$

$-\left[\hat{q}(t)-M(t) C_{2}(t) \hat{y}(t)\right] \overrightarrow{\mathrm{d} W(t)}$.
Now, we set $\hat{q}(t)-M(t) C_{2}(t) \hat{y}(t)=q^{\prime}(t)$, that is, $\hat{q}(t)=M(t) C_{2}(t) \hat{y}(t)+q^{\prime}(t)$, then equation (26) can be written as

$$
\begin{aligned}
-\mathrm{d} \widehat{p}(t) & =\left\{A_{1}^{\top}(t) \widehat{p}(t)+\left[M(t) C_{1}(t)+A_{2}^{\top}(t) M(t) C_{2}(t)\right] \hat{y}(t)+A_{2}^{\top}(t) q^{\prime}(t)\right\} \mathrm{d} t \\
& +\left\{\left[R(t)-C_{2}^{\top}(t) M(t) C_{2}(t)-M(t)\right] \widehat{y}(t)-C_{1}^{\top}(t) \widehat{p}(t)\right. \\
& \left.-C_{2}^{\top}(t) q^{\prime}(t)\right\} \mathrm{d} \overleftarrow{B(}(t)-q^{\prime}(t) \overrightarrow{\mathrm{d} W(t)} .
\end{aligned}
$$


This is a linear backward doubly stochastic differential equation. This is a unique solution $\left(\hat{y}(t), \widehat{p}(t), q^{\prime}(t)\right)$ $\equiv(0,0,0)$, that is, $(\widehat{y}(t), \widehat{p}(t), \widehat{q}(t)) \equiv(0,0,0)$. So, we have $y(t)=G(t) x(t), p(t)=M(t) x(t)$, and $q(t)=N(t) x(t)$.

We complete the proof of Theorem 2 .

Theorem 3. Let the assumptions (A1)-(A3) be satisfied. Assume that $(G(\cdot), M(\cdot), N(\cdot))$ satisfies the generalized Riccati equation, then the optimal control of the delayed doubly stochastic linear quadratic optimal control problem has the following form:

$u^{*}(t)=S^{-1}(t) E^{\mathscr{F}} t\left[F_{1}^{\top}(t+\delta) M(t+\delta)+F_{2}^{\top}(t+\delta) N(t+\delta)\right] x(t+\delta)$,

$$
J\left(u^{*}\right)=-\frac{1}{2}\langle M(0) \eta, \eta\rangle
$$

Proof. From the assumption that $(G(\cdot), M(\cdot), N(\cdot))$ is the solution of the Riccati equation (19) set $y(t)=G(t) x(t)$, $p(t)=M(t) x(t)$, and $q(t)=N(t) x(t)$. Applying Itô's formula to $p(t)$, we have

$$
\begin{aligned}
\mathrm{d} p(t) & =\left[\dot{M}(t) x(t)+M(t) A_{1}(t) x(t)+M(t) C_{1}(t) y(t)+M(t) F_{1}(t) u_{\delta}(t)\right] \mathrm{d} t \\
& +\left[M(t) A_{2}(t) x(t)+M(t) C_{2}(t) y(t)+M(t) F_{2}(t) u_{\delta}(t)\right] \overrightarrow{\mathrm{d} W(t)} \\
& -M(t) y(t) \mathrm{d} \stackrel{\leftarrow}{B}(t), \quad t \in[0, T] .
\end{aligned}
$$

From the definition of the matrix Riccati equation (19), we have

$$
\begin{aligned}
\mathrm{d} p(t)= & \left\{-\left[A_{1}^{\top}(t) M(t)+M(t) A_{1}(t)+M(t) C_{1}(t) G(t)+A_{2}^{\top}(t) N(t)\right.\right. \\
& \left.-K(t)+M(t) F_{1}(t) S^{-1}(t-\delta)\left(F_{1}^{\top}(t) M(t)+F_{2}^{\top}(t) N(t)\right)\right] x(t) \\
& \left.+M(t) A_{1}(t) x(t)+M(t) C_{1}(t) y(t)+M(t) F_{1}(t) u_{\delta}(t)\right\} \mathrm{d} t \\
& +\left\{\left[N(t)-M(t) C_{2}(t) G(t)-M(t) F_{2}(t) S^{-1}(t-\delta)\left(F_{1}^{\top}(t) M(t)\right.\right.\right. \\
& \left.\left.\left.+F_{2}^{\top}(t) N(t)\right)\right] x(t)+M(t) C_{2}(t) y(t)+M(t) F_{2}(t) u_{\delta}(t)\right\} \overrightarrow{\mathrm{d} W(t)} \\
& -\left[R(t) G(t)-C_{1}^{\top}(t) M(t)-C_{2}^{\top}(t) N(t)\right] x(t) \mathrm{d} \overleftarrow{B(}(t), \quad t \in[0, T] .
\end{aligned}
$$

That is,

$$
\begin{aligned}
-\mathrm{d} p(t) & =\left[A_{1}^{\top}(t) p(t)+A_{2}^{\top}(t) q(t)-K(t) x(t)\right] \mathrm{d} t+[R(t) y(t) \\
- & \left.C_{1}^{\top}(t) p(t)-C_{2}^{\top}(t) q(t)\right] \mathrm{d} \overleftarrow{B(}(t)-q(t) \overrightarrow{\mathrm{d} W(t)}, \quad t \in[0, T]
\end{aligned}
$$

So, we know that the solution of system (17) satisfied the formula $\quad y(t)=G(t) x(t), \quad p(t)=M(t) x(t), \quad$ and $q(t)=N(t) x(t)$. Then, the optimal control can be written as (28).
Next, we deduce the objective function $J\left(u^{*}(\cdot)\right)$ by the solution of the Riccati equation and the initial value of the state variable.

Applying Itô's formula to $\langle x(t), p(t)\rangle$ and taking expectation, we have

$$
\begin{aligned}
E[\langle x(T), p(T)\rangle-\langle x(0), p(0)\rangle]= & E[\langle x(T),-Q x(T)\rangle-\langle\eta, M(0) \eta\rangle], \\
E[\langle x(T), p(T)\rangle-\langle x(0), p(0)\rangle]= & E \int_{0}^{T}\left[\langle K(t) x(t), x(t)\rangle+\langle R(t) y(t), y(t)\rangle+\left\langle F_{1}(t) u_{\delta}(t), p(t)\right\rangle+\left\langle F_{2}(t) u_{\delta}(t), q(t)\right\rangle\right] \mathrm{d} t \\
= & E \int_{0}^{T}\left[E^{\mathscr{F}} t\left\langle F_{1}(t+\delta) u(t), p(t+\delta)\right\rangle+E^{\mathscr{F}} t\left\langle F_{2}(t+\delta) u(t), q(t+\delta)\right\rangle+\langle K(t) x(t), x(t)\rangle\right. \\
& +\langle R(t) y(t), y(t)\rangle] \mathrm{d} t .
\end{aligned}
$$


Substituting equalities (33) and (34) into the function $J(u(\cdot))$, we can deduce

$$
\begin{aligned}
J(u(\cdot))= & \frac{1}{2}\left\{E \int_{0}^{T}[\langle K(t) x(t), x(t)\rangle+\langle R(t) y(t), y(t)\rangle\right. \\
& +\langle S(t) u(t), u(t)\rangle] \mathrm{d} t \\
& +\langle Q x(T), x(T)\rangle\} \\
= & -\frac{1}{2}\langle M(0) \eta, \eta\rangle .
\end{aligned}
$$

We complete the proof of Theorem 3.

\section{Applications}

Case 1. For the general delayed doubly stochastic LQ system (1), when the coefficients are

$$
B_{i}(t)=D_{i}(t)=F_{i}(t)=0, \quad i=1,2,
$$

this is the system without time delay. The optimal control deduced by Theorem 1 is consistent with the results in [22].

Case 2. When $F_{i}(t)=0(i=1,2)$ in system (1), this is the case that the system does not contain time delay variables in control variables. The optimal control deduced by Theorem 1 is

$$
u^{*}(t)=S^{-1}(t)\left[E_{1}^{\top}(t) p(t)+E_{2}^{\top}(t) q(t)\right] .
$$

This is consistent with the result in [20]. From the expression of the optimal control, the factors affecting the optimal control are the same as that in [22].

Case 3. For the delayed doubly stochastic LQ system (17), when the optimal control deduced from Theorem 3 is

$$
u^{*}(t)=S^{-1}(t)\left[F_{1}^{\top}(t) M(t)+F_{2}^{\top}(t) N(t)\right] x(t),
$$

the optimal control is derived from the solution of the matrix Riccati equation. This is consistent with the result in [21].

In this paper, we are mainly concerned about the delayed doubly stochastic LQ system in which all the variables contained time delay. We deduced the explicit form of the optimal control for the general case. The results of this paper extend our previous work and enrich the content of the delayed doubly stochastic LQ problem. But, from the conclusion of the article, there are still some contents that need further study. We only discuss the matrix-valued Riccati equation corresponding to the special cases. For a more general Riccati equation of the delayed doubly stochastic LQ system, we will work hard to increase the research on this part in our future work.

\section{Data Availability}

No data were used to support this study.

\section{Conflicts of Interest}

The authors declare that there are no conflicts of interest regarding the publication of this paper.

\section{Acknowledgments}

The authors would like to thank Professor Yuecai Han for valuable suggestions and guidance on this article. This work was supported by science and technology research projects of the Jilin Province Education Department (JJKH20200235KJ) and the National Natural Science Foundation of China (grant number 11871244).

\section{References}

[1] W. Yu, P. Guo, Q. Wang et al., "On a periodic capital injection and barrier dividend strategy in the compound Poisson risk model," Mathematics, vol. 8, no. 4, p. 511, 2020.

[2] W. Yu, Y. Yong, G. Guan, Y. Huang, W. Su, and C. Cui, "Valuing guaranteed minimum death benefits by cosine series expansion," Mathematics, vol. 7, no. 9, p. 835, 2019.

[3] Z. Zhang, Y. Yong, and W. Yu, "Valuing equity-linked death benefits in general exponential Lévy models," Journal of Computational and Applied Mathematics, vol. 365, Article ID 112377, 2020.

[4] W. Yu, F. Wang, Y. Huang, and H. Liu, "Social optimal mean field control problem for population growth model," Asian Journal of Control, pp. 1-8, 2019.

[5] E. Pardoux and S. Peng, "Backward doubly stochastic differential equations and systems of quasilinear SPDEs," Probability Theory and Related Fields, vol. 98, no. 2, pp. 209-227, 1994.

[6] Y. Shi and Q. Zhu, "Partially observed optimal controls of forward-backward doubly stochastic systems," ESAIM: Control, Optimisation and Calculus of Variations, vol. 19, no. 3, pp. 828-843, 2013.

[7] Q. Zhu and Y. Shi, "Optimal control of backward doubly stochastic systems with partial information," IEEE Transactions on Automatic Control, vol. 60, no. 1, pp. 173-178, 2015.

[8] Q. Zhu, Y. Shi, and B. Teng, "Forward-backward doubly stochastic differential equations with random jumps and related games," Asian Journal of Control, 2020.

[9] L. Chen and Z. Wu, "Maximum principle for the stochastic optimal control problem with delay and application," Automatica, vol. 46, no. 6, pp. 1074-1080, 2010.

[10] S. Wu and G. Wang, "Optimal control problem of backward stochastic differential delay equation under partial information," Systems \& Control Letters, vol. 82, no. 6, pp. 71-78, 2015.

[11] S. Lv, R. Tao, and Z. Wu, "Maximum principle for optimal control of anticipated forward-backward stochastic differential delayed systems with regime switching," Optimal Control Applications and Methods, vol. 37, no. 1, pp. 154-175, 2016.

[12] S. Wang and Z. Wu, "Stochastic maximum principle for optimal control problems of forward backward delay systems involving impulse controls," Journal of Systems Science and Complexity, vol. 30, no. 2, pp. 32-58, 2017.

[13] Z. Yu, "The stochastic maximum principle for optimal control problems of delay systems involving continuous and impulse controls," Automatica, vol. 48, no. 10, pp. 2420-2432, 2012.

[14] L. Chen and Z. Wu, "A type of general forward-backward stochastic differential equations and applications," Chinese 
Annals of Mathematics, Series B, vol. 32, no. 2, pp. 279-292, 2011.

[15] H. Tang and $\mathrm{Z}$. Wu, "Stochastic differential equations and stochastic linear quadratic optimal control problem with Lévy processes," Journal of Systems Science and Complexity, vol. 22, no. 1, pp. 122-136, 2009.

[16] J. Huang, X. Li, and J. Shi, "Forward-backward linear quadratic stochastic optimal control problem with delay," Systems \& Control Letters, vol. 61, no. 5, pp. 623-630, 2012.

[17] S. Peng and Z. Yang, "Anticipated backward stochastic differential equations," The Annals of Probability, vol. 37, no. 3, pp. 877-902, 2009.

[18] X. Xu, "Anticipated backward doubly stochastic differential equations," Applied Mathematics and Computation, vol. 220, no. 4, pp. 53-62, 2013.

[19] J. Xu and Y. Han, "Stochastic maximum principle for delayed backward doubly stochastic control systems," The Journal of Nonlinear Sciences and Applications, vol. 10, no. 1, pp. 215226, 2017.

[20] J. Xu, "Stochastic maximum principle for delayed doubly stochastic control systems and their applications," International Journal of Control, vol. 93, no. 6, pp. 1371-1380, 2018.

[21] S. Peng, "Problem of eigenvalues of stochastic Hamiltonian systems with boundary conditions," Stochastic Processes and Their Applications, vol. 88, no. 2, pp. 259-290, 2000.

[22] Y. Han, S. Peng, and Z. Wu, "Maximum principle for backward doubly stochastic control systems with applications," SIAM Journal on Control and Optimization, vol. 48, no. 7, pp. 4224-4241, 2010. 